\title{
Alternative Hosts of Cercospora beticola in Field Surveys and Inoculation Trials
}

\author{
Noel L. Knight, ${ }^{1}$ Niloofar Vaghefi, ${ }^{1}$ Julie R. Kikkert, ${ }^{2}$ and Sarah J. Pethybridge ${ }^{1, \dagger}$ \\ ${ }^{1}$ Plant Pathology and Plant-Microbe Biology Section, School of Integrative Plant Science, Cornell AgriTech at the New York \\ State Agricultural Experiment Station, Cornell University, Geneva, NY 14456 \\ ${ }^{2}$ Cornell Vegetable Program, Cornell Cooperative Extension, Canandaigua, NY 14424
}

\begin{abstract}
Cercospora beticola, the cause of Cercospora leaf spot (CLS) of sugar beet and table beet, has a broad range of potential alternative hosts. The role of these hosts as inoculum sources in the field is unclear and has had limited investigation since the advent of DNA-based pathogen identification. The presence of $C$. beticola on alternative hosts associated with table beet fields of New York was assessed in field surveys during 2016. Lesions were collected, and 71 cercosporoid conidia were isolated for phylogenetic comparison. C. beticola was identified from Solanum ptycanthum $(n=4)$, Chenopodium album $(n=2)$, and Spinacia oleracea $(n=1)$, whereas

C. chenopodii was identified on Chenopodium album $(n=51)$. Artificial inoculation of 21 plants species demonstrated that $C$. beticola was pathogenic to Brassica kaber, Chenopodium album, Carthamus tinctorius, Rumex obtusifolius, and Spinacia oleracea. These results indicate that although $C$. beticola may be pathogenic to a range of plant species, the role of symptomatic tissue for inoculum production on alternative hosts in the field appears limited. Observations of $C$. beticola on necrotic and naturally senescent tissue suggest saprophytic survival on plant debris of a range of species, which has implications for CLS epidemics and disease management.
\end{abstract}

A comprehensive knowledge of potential sources of inoculum for epidemic initiation is vital for the design of effective disease management strategies. Cercospora beticola Sacc., the cause of the foliar disease Cercospora leaf spot (CLS) on sugar beet and table beet (Beta vulgaris subsp. vulgaris L.) (Weiland and Koch 2004), survives on alternative hosts and colonized plant debris, which are believed to be the primary sources of inoculum for CLS epidemics (Jacobsen and Franc 2009; Khan et al. 2008; McKay and Pool 1918; Pool and McKay 1916; Solel 1970). External sources of inoculum, such as infested beet seed, have also been suggested to harbor $C$. beticola inoculum (Knight et al. 2018; Vaghefi et al. 2017); however, direct evidence is limited (McKay and Pool 1918). The ability of C. beticola to colonize a broad range of alternative hosts plays an important role in the disease cycle of CLS. Potential alternative hosts for $C$. beticola include crop and weed species, such as sugar beet, table beet, fodder beet, Swiss chard (B. vulgaris subsp. vulgaris) (McKay and Pool 1918; Vestal 1933), B. vulgaris subsp. maritima (Jacobsen and Franc 2009), safflower (Carthamus tinctorius) (Lartey et al. 2005), lettuce (Lactuca sativa) (Hotegni Houessou et al. 2011), and spinach (Spinacia oleracea) (Vestal 1933), along with species in the genera Acanthus, Amaranthus, Apium, Atriplex, Chenopodium, Chrysanthemum, Cycloloma, Goniolimon, Limonium, Malva, and Plantago (Bobev et al. 2009; Groenewald et al. 2006; Jacobsen and Franc 2009; Rooney-Latham et al. 2011).

Current address of N. Vaghefi: Centre for Crop Health, University of Southern Queensland, Toowoomba, QLD 4350, Australia

${ }^{\dagger}$ Corresponding author: S. J. Pethybridge; sjp277@cornell.edu

Funding: This research was supported by the U.S. Department of Agriculture National Institute of Food and Agriculture Hatch project NYG-625424 and the Federal Capacity Funds Initiatives (2015-16-118 and 2018-19-113) managed by Cornell AgriTech at the New York State Agricultural Experiment Station, Cornell University, Geneva, New York.

*The $\boldsymbol{e}$-Xtra logo stands for "electronic extra" and indicates that four supplementary tables are published online.

The author(s) declare no conflict of interest.

Accepted for publication 13 March 2019.

C 2019 The American Phytopathological Society
Investigations of the host range of $C$. beticola based on pathogenicity trials have yielded conflicting results (McKay and Pool 1918; Vestal 1933), whereas other studies have implied potential hosts based on isolation of $C$. beticola without pathogenicity assessment (Groenewald et al. 2006, 2013). For example, evaluations of the pathogenicity of $C$. beticola to lettuce have either reported $50 \%$ disease incidence (Vestal 1933) or described lettuce as a nonhost (McKay and Pool 1918). Additionally, pathogenicity of C. beticola on lambsquarters (Chenopodium album) was reported (Nagel 1932 as cited in Vestal 1933) but paradoxically declared a nonhost by others (McKay and Pool 1918; Schmidt 1928 as cited in Vestal 1933). Lambsquarters is a weed common to table beet fields in New York and is frequently found with CLS-like symptoms, leading to the assumption that it was a dominant source of inoculum contributing to CLS epidemics (Vaghefi et al. 2017). However, investigation of these symptoms on lambsquarters growing within table beet fields in New York indicated that $C$. chenopodii is the predominantly associated species (Vaghefi et al. 2017). This emphasizes the importance of comprehensive surveys of leaf spots associated with plants in table beet fields for investigating the prevalence and incidence of $C$. beticola on species other than B. vulgaris.

The foundational work on the host range of C. beticola (McKay and Pool 1918; Vestal 1933) was performed prior to the molecular characterization of $C$. beticola (Groenewald et al. 2005, 2006). Traditionally, morphological characteristics and host range were used to describe and identify Cercospora species (Chupp 1954); however, the identification of clades within the morphologically indistinguishable $C$. apii complex using DNA sequences of multiple phylogenetically informative loci has modified species boundaries and classifications (Bakhshi et al. 2015b; Cai et al. 2011; Crous et al. 2015; Groenewald et al. 2005). For example, phylogenetic assessments have suggested the separation of $C$. beticola from morphologically indistinguishable species such as $C$. apii and $C$. apiicola (Groenewald et al. 2005, 2006). Therefore, the accuracy of species identification in studies without DNA sequencing is uncertain, and investigation of the traditionally reported host range requires $C$. beticola isolates identified using DNA sequence-based techniques.

Understanding the host range of $C$. beticola is a priority for CLS disease management in table beet fields in New York. The survival of $C$. beticola on alternative hosts may contribute to the initiation of CLS epidemics in table beet fields and may affect the genetic diversity of $C$. beticola populations by disrupting genetic drift and selection pressures. The aim of this study was to determine the frequency of $C$. beticola associated with foliar disease of potential 
Table 1. Cercospora isolates collected in field surveys during 2016 in New York and reference isolates included for phylogenetic comparisons ${ }^{\text {a }}$

\begin{tabular}{|c|c|c|c|c|c|c|c|c|}
\hline \multirow[b]{2}{*}{ Species } & \multirow[b]{2}{*}{ Isolate } & \multirow[b]{2}{*}{ Host } & \multirow[b]{2}{*}{ Source } & \multicolumn{5}{|c|}{ GenBank accession number } \\
\hline & & & & act & cmdA & hisH3 & ITS & tef $1-\alpha$ \\
\hline C. beticola & CBS 116456; CPC 11557 & Beta vulgaris & Italy & AY840458 & AY840425 & AY840392 & AY840527 & AY840494 \\
\hline C. beticola & CBS 117.47 & B. vulgaris & $\begin{array}{l}\text { Czech } \\
\text { Republic }\end{array}$ & DQ233374 & DQ233400 & DQ233426 & DQ233322 & DQ233348 \\
\hline C. beticola & ICMP 21691; Tb14-081 & B. vulgaris subsp. vulgaris & USA & MF680889 & MF681401 & MF680991 & MF681093 & MF681195 \\
\hline C. beticola & ICMP 21692; Tb14-085 & B. vulgaris subsp. vulgaris & USA & MF680890 & MF681402 & MF680992 & MF681094 & MF681196 \\
\hline C. beticola & ICMP 21693; Tb14-140 & B. vulgaris subsp. vulgaris & USA & MF680894 & MF681406 & MF680996 & MF681098 & MF681200 \\
\hline C. beticola & ICMP 21694; Tb15-259 & B. vulgaris subsp. vulgaris & USA & MF680942 & MF681454 & MF681044 & MF681146 & MF681248 \\
\hline C. beticola & Lq16-006 & Chenopodium album & Field 8, NY & MK272177 & MK272269 & MK271917 & MK271993 & MK272129 \\
\hline C. beticola & Lq16-010 & Chenopodium album & Field 9, NY & MK272181 & MK272273 & MK271921 & MK271997 & MK272130 \\
\hline C. beticola & Ns16-001 & Solanum ptycanthum & Field 3, NY & MK272225 & MK272317 & MK271963 & MK272041 & MK272133 \\
\hline C. beticola & Ns16-002 & Solanum ptycanthum & Field 3, NY & MK272226 & MK272318 & MK271883 & MK272042 & MK272134 \\
\hline C. beticola & Ns16-005 & Solanum ptycanthum & Field 3, NY & MK272229 & MK272321 & MK271964 & MK272045 & MK272137 \\
\hline C. beticola & Ns16-006 & Solanum ptycanthum & Field 3, NY & MK272230 & MK272322 & MK271965 & MK272046 & MK272138 \\
\hline C. beticola & Sp16-004 & Spinacia oleracea & Field 2, NY & MK272243 & MK272335 & MK271970 & MK272059 & MK272151 \\
\hline C. cf. flagellaris & CBS 132646; CPC 10681 & Cichorium intybus & South Korea & JX143113 & JX142867 & $J X 142621$ & JX143601 & JX143359 \\
\hline C. cf. flagellaris & CBS 132670; CPC 14487 & Sigesbeckia pubescens & South Korea & JX143117 & JX142871 & JX142625 & JX143605 & JX143363 \\
\hline C. cf. flagellaris & ICMP 21686; Tb15-020 & B. vulgaris subsp. vulgaris & USA & MF680899 & MF681411 & MF681001 & MF681103 & MF681205 \\
\hline C. cf. flagellaris & Ns16-003 & Solanum ptycanthum & Field 3, NY & MK272227 & MK272319 & MK271884 & MK272043 & MK272135 \\
\hline C. cf. flagellaris & Ns16-004 & Solanum ptycanthum & Field 3, NY & MK272228 & MK272320 & MK271885 & MK272044 & MK272136 \\
\hline C. cf. flagellaris & $\operatorname{Pg} 16-001$ & Amaranthus retroflexus & Field 5, NY & MK272231 & MK272323 & MK271886 & MK272047 & MK272139 \\
\hline C. cf. flagellaris & Pg16-002 & A. retroflexus & Field 5, NY & MK272232 & MK272324 & MK271887 & MK272048 & MK272140 \\
\hline C. cf. flagellaris & Pg16-003 & A. retroflexus & Field 5, NY & MK272233 & MK272325 & MK271888 & MK272049 & MK272141 \\
\hline C. cf. flagellaris & Sp16-001 & Spinacia oleracea & Field 2, NY & MK272240 & MK272332 & MK271891 & MK272056 & MK272148 \\
\hline C. cf. flagellaris & Sp16-002 & Spinacia oleracea & Field 2, NY & MK272241 & MK272333 & MK271892 & MK272057 & MK272149 \\
\hline C. cf. flagellaris & Sp16-003 & Spinacia oleracea & Field 2, NY & MK272242 & MK272334 & MK271893 & MK272058 & MK272150 \\
\hline C. cf. flagellaris & Sp16-005 & Spinacia oleracea & Field 2, NY & MK272244 & MK272336 & MK271894 & MK272060 & MK272152 \\
\hline C. cf. flagellaris & Sp16-006 & Spinacia oleracea & Field 2, NY & MK272245 & MK272337 & MK271895 & MK272061 & MK272153 \\
\hline C. cf. flagellaris & Sp16-007 & Spinacia oleracea & Field 2, NY & MK272246 & MK272338 & MK271896 & MK272062 & MK272154 \\
\hline C. chenopodii & CBS 132620; CPC 14237 & Chenopodium album & France & JX143081 & JX142835 & JX142589 & JX143571 & JX143327 \\
\hline C. chenopodii & CCTU 1033 & Chenopodium album & Iran & KJ885954 & KJ885793 & KJ886115 & KJJ886437 & KJ886276 \\
\hline C. chenopodii & CCTU 1060 & Chenopodium album & Iran & KJ885955 & KJ885794 & KJ886116 & KJ886438 & KJ886277 \\
\hline C. chenopodii & CPC 15763 & Chenopodium sp. & Mexico & JX143085 & JX142839 & JX142593 & JX143575 & JX143331 \\
\hline C. chenopodii & Lq16-001 & Chenopodium album & Field 2, NY & MK272172 & MK272264 & MK271914 & MK271988 & MK272080 \\
\hline C. chenopodii & Lq16-002; ICMP 22619 & Chenopodium album & Field 2, NY & MK272173 & MK272265 & MK271915 & MK271989 & MK272081 \\
\hline C. chenopodii & Lq16-003 & Chenopodium album & Field 4, NY & MK272174 & MK272266 & MK271879 & MK271990 & MK272127 \\
\hline C. chenopodii & Lq16-004 & Chenopodium album & Field 4, NY & MK272175 & MK272267 & MK271880 & MK271991 & MK272128 \\
\hline C. chenopodii & Lq16-005 & Chenopodium album & Field 4, NY & MK272176 & MK272268 & MK271916 & MK271992 & MK272082 \\
\hline C. chenopodii & Lq16-007 & Chenopodium album & Field $8, \mathrm{NY}$ & MK272178 & MK272270 & MK271918 & MK271994 & MK272083 \\
\hline C. chenopodii & Lq16-008 & Chenopodium album & Field $8, \mathrm{NY}$ & MK272179 & MK272271 & MK271919 & MK271995 & MK272084 \\
\hline C. chenopodii & Lq16-009 & Chenopodium album & Field $8, N Y$ & MK272180 & MK272272 & MK271920 & MK271996 & MK272085 \\
\hline C. chenopodii & Lq16-011 & Chenopodium album & Field $10, \mathrm{NY}$ & MK272182 & MK272274 & MK271922 & MK271998 & MK272086 \\
\hline C. chenopodii & Lq16-012 & Chenopodium album & Field $10, \mathrm{NY}$ & MK272183 & MK272275 & MK271923 & MK271999 & MK272087 \\
\hline C. chenopodii & Lq16-013 & Chenopodium album & Field $10, \mathrm{NY}$ & MK272184 & MK272276 & MK271924 & MK272000 & MK272088 \\
\hline C. chenopodii & Lq16-014 & Chenopodium album & Field $10, \mathrm{NY}$ & MK272185 & MK272277 & MK271925 & MK272001 & MK272089 \\
\hline C. chenopodii & Lq16-015 & Chenopodium album & Field 5, NY & MK272186 & MK272278 & MK271926 & MK272002 & MK272090 \\
\hline C. chenopodii & Lq16-016 & Chenopodium album & Field 5, NY & MK272187 & MK272279 & MK271927 & MK272003 & MK272091 \\
\hline C. chenopodii & Lq16-017 & Chenopodium album & Field 5, NY & MK272188 & MK272280 & MK271928 & MK272004 & MK272092 \\
\hline C. chenopodii & Lq16-018 & Chenopodium album & Field $2, \mathrm{NY}$ & MK272189 & MK272281 & MK271929 & MK272005 & MK272093 \\
\hline C. chenopodii & Lq16-019 & Chenopodium album & Field 2, NY & MK272190 & MK272282 & MK271930 & MK272006 & MK272094 \\
\hline C. chenopodii & Lq16-020 & Chenopodium album & Field 2, NY & MK272191 & MK272283 & MK271931 & MK272007 & MK272095 \\
\hline C. chenopodii & Lq16-021 & Chenopodium album & Field 2, NY & MK272192 & MK272284 & MK271932 & MK272008 & MK272096 \\
\hline C. chenopodii & Lq16-022 & Chenopodium album & Field 2, NY & MK272193 & MK272285 & MK271933 & MK272009 & MK272097 \\
\hline C. chenopodii & Lq16-024 & Chenopodium album & Field 3, NY & MK272194 & MK272286 & MK271934 & MK272010 & MK272098 \\
\hline C. chenopodii & Lq16-025 & Chenopodium album & Field 3, NY & MK272195 & MK272287 & MK271935 & MK272011 & MK272099 \\
\hline C. chenopodii & Lq16-027 & Chenopodium album & Field $3, \mathrm{NY}$ & MK272196 & MK272288 & MK271936 & MK272012 & MK272100 \\
\hline C. chenopodii & Lq16-028 & Chenopodium album & Field 3, NY & MK272197 & MK272289 & MK271937 & MK272013 & MK272101 \\
\hline C. chenopodii & Lq16-029 & Chenopodium album & Field 3, NY & MK272198 & MK272290 & MK271938 & MK272014 & MK272102 \\
\hline C. chenopodii & Lq16-030 & Chenopodium album & Field 3, NY & MK272199 & MK272291 & MK271881 & MK272015 & MK272131 \\
\hline C. chenopodii & Lq16-031 & Chenopodium album & Field $3, \mathrm{NY}$ & MK272200 & MK272292 & MK271939 & MK272016 & MK272103 \\
\hline C. chenopodii & Lq16-032 & Chenopodium album & Field 3, NY & MK272201 & MK272293 & MK271940 & MK272017 & MK272104 \\
\hline C. chenopodii & Lq16-033 & Chenopodium album & Field 3, NY & MK272202 & MK272294 & MK271941 & MK272018 & MK272105 \\
\hline C. chenopodii & Lq16-034 & Chenopodium album & Field 3, NY & MK272203 & MK272295 & MK271942 & MK272019 & MK272106 \\
\hline C. chenopodii & Lq16-035 & Chenopodium album & Field 3, NY & MK272204 & MK272296 & MK271943 & MK272020 & MK272107 \\
\hline C. chenopodii & Lq16-036 & Chenopodium album & Field 3, NY & MK272205 & MK272297 & MK271882 & MK272021 & MK272132 \\
\hline & & & & & & & (Continued o & n next page) \\
\hline
\end{tabular}

\footnotetext{
${ }^{a}$ Reference isolates are indicated in bold. $a c t=$ actin, $c m d A=$ calmodulin, his $H 3=$ histone, ITS $=$ internal transcribed spacer, and tefl- $\alpha=$ translation elongation
} factor 1-alpha. 
alternative hosts in table beet fields of New York and to investigate the host range of $C$. beticola across selected crop and weed species using pathogenicity testing.

\section{Materials and Methods}

Field surveys and isolations. Leaves of weed and crop species with foliar symptoms as either CLS-like spots or necrotic lesions were sampled opportunistically from 10 fields during September 2016 (Supplementary Table S1). Fields included broad-acre and mixed-cropping systems that had grown table beet in 2014, 2015, or 2016 (Supplementary Table S2). Sampling of weed and crop species varied according to in-field frequency and the incidence and severity of disease.

Following sampling, all lesions on a plant were inspected for cercosporoid conidia under $63 \times$ magnification using a SZX10 stereo microscope (Olympus, Tokyo, Japan) with a KL 1600 LED light source (Schott, Elmsford, NY). Single-conidium cultures for individual lesions were produced using the method described by Knight et al. (2018), with the exception of lambsquarters. When numerous lesions were present on diseased lambsquarters, 10 to 20 lesions were randomly selected for morphological observation of conidia. Conidia exhibiting morphology similar to C. beticola (Jacobsen and Franc 2009) were transferred to $2 \%$ water agar (20 g/liter; Hardy Diagnostics, Santa Maria, CA).

DNA extraction, PCR, and sequencing. Mycelial growth and DNA extraction were performed as described in Knight et al. (2018). DNA of isolates from field surveys (Table 1) was amplified using five primer pairs (Supplementary Table S3) previously described in phylogenetic studies of Cercospora species (Bakhshi et al. 2018; Groenewald et al. 2005, 2013; Vaghefi et al. 2018). The primer pairs amplified partial sequences of internal transcribed spacers of the nuclear ribosomal DNA including the 5.8S region (ITS), actin (act), calmodulin ( $c m d A)$, histone (hisH3), and translation elongation factor 1-alpha (tefl- $\alpha)$ genes. PCR was performed as described by Groenewald et al. (2005) using a T100 Thermal Cycler (Bio-Rad, Hercules, CA). PCR products were visualized after electrophoresis on a $10 \mathrm{~g} /$ liter agarose gel in Tris-acetate-EDTA containing $0.001 \%$ (vol/vol) GelRed (Biotium Inc., Hayward, CA). Product size was determined against a 100-bp DNA ladder (Axygen Inc., Union City, CA). PCR products were subsequently treated with exonuclease I and shrimp alkaline phosphatase (New England BioLabs, Ipswich, MA) and were Sanger sequenced at the Genomics Facility at the Cornell University Institute of Biotechnology (Ithaca, NY) with the forward and reverse primers used for the original PCR. Consensus sequences were obtained by pairwise alignment of forward and reverse sequences in Geneious version 11.1.5 (https://www.geneious.com/; Kearse et al. 2012). Sequences were deposited in the GenBank nucleotide database (Table 1).

Phylogenetic analyses. Sequences of reference isolates were obtained from the National Center for Biotechnology Information nucleotide database (Table 1) and aligned with DNA sequences of the field isolates. Multiple sequence alignments for each locus were conducted using MAFFT version 7.388 (Katoh and Standley 2013; Katoh et al. 2002) in Geneious. Sequences of the five loci were subsequently concatenated as "act $+c m d A+h i s H 3+$ ITS + tefl- $\alpha$ " and phylogenetic trees were generated using the approximately maximum likelihood method of FastTree version 2.1.5 (Price et al. 2009, 2010) in Geneious. Node support values were computed using the ShimodairaHasegawa test (Shimodaira and Hasegawa 1999) in the FastTree plugin in Geneious. Alignments and phylogenetic trees were deposited in TreeBASE (https://www.treebase.org/ and http://purl.org/phylo/treebase/phylows/study/TB2:S23738).

C. beticola isolates and inoculum preparation. Two isolates of C. beticola (Tb14-081 [ICMP:21691] and Tb14-085 [ICMP:21692]) from CLS of table beet in New York, representing two clades within C. beticola (Vaghefi et al. 2018), were selected for inoculation experiments. Inoculum was prepared according to Secor and Rivera (2012) with slight modifications. Briefly, $10 \mathrm{ml}$ of sterile distilled water was added to a 2-week-old culture grown on potato dextrose agar (Hardy Diagnostics), and mycelium was disrupted from the surface using the edge of a microscope slide. Threehundred microliters of the mycelial suspension was spread onto each of 20 clarified V8 agar plates $(10 \%$ [vol/vol] clarified V8 broth, 20 g/liter agar) (Miller 1955; Secor and Rivera 2012). The plates were subsequently dried for $30 \mathrm{~min}$ in a laminar flow cabinet, sealed with Parafilm (Bemis Co., Neenah, WI), and incubated for 6 days with an 8-h photoperiod. Conidia were collected by flooding plates with $5 \mathrm{ml}$ of sterile distilled water and disrupting the surface with a glass spreader before filtering through two layers of cheesecloth. The concentration of the conidial suspension was quantified using

Table 1. (Continued from previous page)

\begin{tabular}{|c|c|c|c|c|c|c|c|c|}
\hline \multirow[b]{2}{*}{ Species } & \multirow[b]{2}{*}{ Isolate } & \multirow[b]{2}{*}{ Host } & \multirow[b]{2}{*}{ Source } & \multicolumn{5}{|c|}{ GenBank accession number } \\
\hline & & & & act & cmdA & hisH3 & ITS & tefl- $\alpha$ \\
\hline C. chenopodii & Lq16-037 & Chenopodium album & Field 3, NY & MK272206 & MK272298 & MK271944 & MK272022 & MK272108 \\
\hline C. chenopodii & Lq16-038 & Chenopodium album & Field 3, NY & MK272207 & MK272299 & MK271945 & MK272023 & MK272109 \\
\hline C. chenopodii & Lq16-039 & Chenopodium album & Field 7, NY & MK272208 & MK272300 & MK271946 & MK272024 & MK272110 \\
\hline C. chenopodii & Lq16-042 & Chenopodium album & Field 7, NY & MK272209 & MK272301 & MK271947 & MK272025 & MK272111 \\
\hline C. chenopodii & Lq16-043 & Chenopodium album & Field 7, NY & MK272210 & MK272302 & MK271948 & MK272026 & MK272112 \\
\hline C. chenopodii & Lq16-044 & Chenopodium album & Field 7, NY & MK272211 & MK272303 & MK271949 & MK272027 & MK272113 \\
\hline C. chenopodii & Lq16-045 & Chenopodium album & Field 7, NY & MK272212 & MK272304 & MK271950 & MK272028 & MK272114 \\
\hline C. chenopodii & Lq16-046 & Chenopodium album & Field 7, NY & MK272213 & MK272305 & MK271951 & MK272029 & MK272115 \\
\hline C. chenopodii & Lq16-047 & Chenopodium album & Field 7, NY & MK272214 & MK272306 & MK271952 & MK272030 & MK272116 \\
\hline C. chenopodii & Lq16-048 & Chenopodium album & Field 7, NY & MK272215 & MK272307 & MK271953 & MK272031 & MK272117 \\
\hline C. chenopodii & Lq16-049 & Chenopodium album & Field 7, NY & MK272216 & MK272308 & MK271954 & MK272032 & MK272118 \\
\hline C. chenopodii & Lq16-050 & Chenopodium album & Field 7, NY & MK272217 & MK272309 & MK271955 & MK272033 & MK272119 \\
\hline C. chenopodii & Lq16-051 & Chenopodium album & Field 7, NY & MK272218 & MK272310 & MK271956 & MK272034 & MK272120 \\
\hline C. chenopodii & Lq16-052 & Chenopodium album & Field 7, NY & MK272219 & MK272311 & MK271957 & MK272035 & MK272121 \\
\hline C. chenopodii & Lq16-053 & Chenopodium album & Field 7, NY & MK272220 & MK272312 & MK271958 & MK272036 & MK272122 \\
\hline C. chenopodii & Lq16-054 & Chenopodium album & Field 7, NY & MK272221 & MK272313 & MK271959 & MK272037 & MK272123 \\
\hline C. chenopodii & Lq16-055 & Chenopodium album & Field 7, NY & MK272222 & MK272314 & MK271960 & MK272038 & MK272124 \\
\hline C. chenopodii & Lq16-056 & Chenopodium album & Field 7, NY & MK272223 & MK272315 & MK271961 & MK272039 & MK272125 \\
\hline C. chenopodii & Lq16-057 & Chenopodium album & Field 7, NY & MK272224 & MK272316 & MK271962 & MK272040 & MK272126 \\
\hline Cercospora sp. G & CPC 5438 & Salvia viscosa & New Zealand & JX143196 & JX142950 & JX142704 & JX143682 & JX143442 \\
\hline Cercospora sp. G & ICMP 21688; Tb15-004 & B. vulgaris subsp. vulgaris & USA & MF680897 & MF681409 & MF680999 & MF681101 & MF681203 \\
\hline Cercospora sp. G & Pg16-004 & A. retroflexus & Field 7, NY & MK272234 & MK272326 & MK271889 & MK272050 & MK272142 \\
\hline Cercospora sp. G & Pg16-005 & A. retroflexus & Field 7, NY & MK272235 & MK272327 & MK271890 & MK272051 & MK272143 \\
\hline C. zeae-maydis & CBS 117756; A359 & Zea mays & USA & DQ185097 & DQ185109 & DQ185121 & DQ185073 & DQ185085 \\
\hline
\end{tabular}


a hemocytometer (Hausser Scientific, Horsham, PA) and diluted to $10^{5}$ conidia/ml. Polysorbate-20 (as $0.01 \%$ [ $\mathrm{vol} / \mathrm{vol}$ ] Tween-20; Agdia, Elkhart, IN) was added to the suspension. The viability of conidia was assessed by spreading $100 \mu \mathrm{l}$ of the suspension onto $2 \%$ water agar (Hardy Diagnostics) and counting germinated conidia $(n=50)$ at $24 \mathrm{~h}$. Conidia were considered germinated when the length of the germ tube was at least the length of the conidium.

Plant species, growth conditions, and inoculation. Eight- to 10week-old plants of 21 species (Table 2) were inoculated with each of the $C$. beticola isolates. Seeds of each species (Supplementary Table S4) were planted at a depth of $1 \mathrm{~cm}$ in soil medium, SunshineMix No. 8 Fafard-2 (Sungro Horticulture, Agawam, MA), in round pots $(15 \mathrm{~cm}$ diameter $\times 13 \mathrm{~cm} \mathrm{depth})$. Glasshouse conditions were maintained at $21^{\circ} \mathrm{C} / 15^{\circ} \mathrm{C}$ for $14-\mathrm{h} / 10$-h light/dark photoperiod cycles. Germination of eastern black nightshade (Solanum ptycanthum) and dock weed (Rumex obtusifolius) seeds was induced as described by Zhou et al. (2005). Briefly, seeds were soaked in distilled water at $20^{\circ} \mathrm{C}$ in the dark for $24 \mathrm{~h}$, then placed on filter paper in a Petri plate moistened with $2 \mathrm{ml}$ of distilled water. Plates were incubated under a 14-h/10-h light/dark photoperiod, with $30^{\circ} \mathrm{C} / 25^{\circ} \mathrm{C}$ alternating temperatures, until germination (approximately 7 days). Germinated seeds were placed in the soil medium at a depth of $1 \mathrm{~cm}$ and then placed in the glasshouse.

Three inoculation treatments were applied in each trial, including C. beticola isolates Tb14-081 and Tb14-085 and a negative control solution $(0.01 \%$ [ $\mathrm{vol} / \mathrm{vol}]$ polysorbate-20). Treatments were applied

Table 2. Disease incidence, symptom type, and reisolation frequencies following inoculation of 21 plant species with two isolates of Cercospora beticola from Cercospora leaf spot in table beet

\begin{tabular}{|c|c|c|c|c|c|c|c|}
\hline Common name & Species & Cultivar ${ }^{\mathrm{a}}$ & Isolate & Lesions $(\%)^{\mathbf{b}}$ & $\begin{array}{c}\text { Marginal } \\
\text { necrosis }(\%)^{\mathbf{c}}\end{array}$ & $\begin{array}{c}\text { Symptomless } \\
(\%)^{\mathrm{d}}\end{array}$ & $\begin{array}{c}\text { Reisolation } \\
(\%)^{\mathrm{e}}\end{array}$ \\
\hline \multirow{2}{*}{ Broadleaf plantain } & Plantago major & - & Tb14-081 & 10 & 40 & 60 & 40 \\
\hline & & & Tb14-085 & 20 & 80 & 10 & 90 \\
\hline \multirow[t]{2}{*}{ Buckwheat } & Fagopyrum esculentum & - & Tb14-081 & 0 & 100 & 0 & 80 \\
\hline & & & Tb14-085 & 0 & 100 & 0 & 70 \\
\hline \multirow[t]{2}{*}{ Carrot } & Daucus carota subsp. sativus & Carson & Tb14-081 & 0 & 50 & 50 & 60 \\
\hline & & & Tb14-085 & 0 & 60 & 40 & 60 \\
\hline \multirow[t]{2}{*}{ Celery } & $\begin{array}{l}\text { Apium graveolens } \\
\text { var. secalinum }\end{array}$ & Cutting & Tb14-081 & 0 & 0 & 100 & 0 \\
\hline & & & Tb14-085 & 0 & 0 & 100 & 0 \\
\hline \multirow[t]{2}{*}{ Dock weed } & Rumex obtusifolius & - & Tb14-081 & 100 & 0 & 0 & 90 \\
\hline & & & Tb14-085 & 100 & 0 & 0 & 90 \\
\hline \multirow{2}{*}{$\begin{array}{l}\text { Eastern black } \\
\text { nightshade }\end{array}$} & Solanum ptycanthum & - & Tb14-081 & 30 & 40 & 30 & 60 \\
\hline & & & Tb14-085 & 0 & 60 & 40 & 80 \\
\hline \multirow[t]{2}{*}{ Lambsquarters } & Chenopodium album & - & Tb14-081 & 100 & 0 & 0 & 100 \\
\hline & & & Tb14-085 & 100 & 0 & 0 & 100 \\
\hline \multirow[t]{2}{*}{ Lettuce } & Lactuca sativa & Great Lakes & Tb14-081 & 0 & 100 & 0 & 80 \\
\hline & & & Tb14-085 & 0 & 100 & 0 & 90 \\
\hline \multirow[t]{2}{*}{ Lucerne } & Medicago sativa & Summer & Tb14-081 & 0 & 30 & 70 & 30 \\
\hline & & & Tb14-085 & 0 & 20 & 80 & 20 \\
\hline \multirow[t]{2}{*}{ Pea } & Pisum sativum & Bolero & Tb14-081 & 0 & 0 & 100 & 0 \\
\hline & & & Tb14-085 & 0 & 0 & 100 & 0 \\
\hline \multirow[t]{2}{*}{ Pigweed } & Amaranthus retroflexus & - & Tb14-081 & 10 & 0 & 90 & 10 \\
\hline & & & Tb14-085 & 0 & 0 & 100 & 0 \\
\hline \multirow[t]{2}{*}{ Ragweed } & Ambrosia artemisiifolia & - & Tb14-081 & 0 & 0 & 100 & 0 \\
\hline & & & Tb14-085 & 0 & 0 & 100 & 0 \\
\hline \multirow[t]{2}{*}{ Rye } & Secale cereale & Aroostook & Tb14-081 & 0 & 0 & 100 & 0 \\
\hline & & & Tb14-085 & 0 & 20 & 30 & 20 \\
\hline \multirow[t]{2}{*}{ Safflower } & Carthamus tinctorius & Centennial & Tb14-081 & 100 & 0 & 0 & 100 \\
\hline & & & Tb14-085 & 100 & 0 & 0 & 100 \\
\hline \multirow[t]{2}{*}{ Snap bean } & Phaseolus vulgaris & Huntington & Tb14-081 & 0 & 60 & 40 & 50 \\
\hline & & & Tb14-085 & 20 & 60 & 20 & 70 \\
\hline \multirow[t]{2}{*}{ Spinach } & Spinacia oleracea & Bloomsdale & Tb14-081 & 100 & 0 & 0 & 100 \\
\hline & & & Tb14-085 & 100 & 0 & 0 & 100 \\
\hline \multirow[t]{2}{*}{ Sweet potato } & Ipomoea batatas & Beauregard & Tb14-081 & 0 & 0 & 100 & 0 \\
\hline & & & Tb14-085 & 0 & 0 & 100 & 0 \\
\hline \multirow[t]{2}{*}{ Velvetleaf } & Abutilon theophrasti & - & Tb14-081 & 0 & 0 & 100 & 0 \\
\hline & & & Tb14-085 & 0 & 0 & 100 & 0 \\
\hline \multirow[t]{2}{*}{ Watermelon } & Citrullus vulgaris var. lanatus & Crimson Sweet & Tb14-081 & 0 & 100 & 0 & 70 \\
\hline & & & Tb14-085 & 0 & 100 & 0 & 100 \\
\hline \multirow[t]{2}{*}{ Wild mustard } & Brassica kaber & - & Tb14-081 & 100 & 100 & 0 & 60 \\
\hline & & & Tb14-085 & 100 & 100 & 0 & 70 \\
\hline \multirow[t]{2}{*}{ Yellow nutsedge } & Cyperus esculentus & - & Tb14-081 & 0 & 0 & 100 & 0 \\
\hline & & & Tb14-085 & 0 & 0 & 100 & 0 \\
\hline
\end{tabular}

a Dashes indicate species that did not have cultivars.

${ }^{\mathrm{b}}$ Lesions indicate the presence of circular-shaped necrotic tissue after inoculation with C. beticola. The percentage incidence of lesions was calculated by dividing the total number of symptomatic plants by the total number of plants, multiplied by 100 .

${ }^{\mathrm{c}}$ Marginal necrosis indicates the presence of necrotic leaf edges after inoculation with $C$. beticola. The percentage incidence of marginal necrosis was calculated by dividing the total number of symptomatic plants by the total number of plants, multiplied by 100 .

d Symptomless indicates the absence of lesions or necrotic leaf edges after inoculation with $C$. beticola. The percentage incidence of symptomless plants was calculated by dividing the total number of symptomless plants by the total number of plants, multiplied by 100 .

${ }^{\mathrm{e}}$ Reisolation indicates the observation of stromata and conidia with morphology resembling $C$. beticola. The reisolation frequency was calculated by dividing the number of plants with isolations resulting in $C$. beticola by the total number of plants, multiplied by 100. 
to five plants of three to four species, along with five table beet plants (cultivar Ruby Queen) as positive controls for CLS. For each treatment, plants were arranged in a randomized block design on separate benches. Inoculation of each plant species and fungal isolate was replicated.

Prior to inoculation, each potted plant was placed in a transparent 32-liter plastic bag (ULINE, Pleasant Prairie, WI) in a growth chamber. The adaxial leaf surface of each plant was inoculated with approximately $12 \mathrm{ml}$ of inoculum using a 355-ml TS-12 trigger pump sprayer (Sprayco, Livonia, MI) and was sealed in the plastic bag. After $48 \mathrm{~h}$, the plastic bags were removed, and a misting apparatus was turned on for $4 \mathrm{~h}$ on 5 days each week for 4 weeks. Growth chamber conditions were $25^{\circ} \mathrm{C}$ with $16 \mathrm{~h}$ of light at 820 lux $(110 \mathrm{~W}$ fluorescent mercury bulbs; Philips, Andover, MA) and a mist water temperature of $22.7^{\circ} \mathrm{C}$. Relative humidity was maintained at $>90 \%$ for $4 \mathrm{~h}$ per day.

C. beticola pathogenicity. After inoculation, plants were examined for disease symptoms every second day for 4 weeks. Symptomatic tissues were collected, evaluated under $63 \times$ magnification using a SZX10 stereo microscope (Olympus) with a KL 1600 LED light source (Schott), and incubated for $24 \mathrm{~h}$ in a plastic box containing moist paper. When cercosporoid conidia were observed, $20 \mu \mathrm{l}$ of sterile distilled water was pipetted onto the symptomatic tissue, conidia were dislodged with the pipette tip, and the suspension was transferred to a microscope slide (VWR, Radnor, PA) for examination under 200 to $400 \times$ magnification using a BX53 compound microscope (Olympus). Leaf tissues with conidia resembling the expected morphology and dimensions of $C$. beticola were considered a positive reisolation. A maximum of five isolations per plant were conducted. The disease incidence (number of symptomatic plants divided by total number of plants $\times 100)$ and reisolation frequency (number of plants with isolations resulting in C. beticola divided by total number of plants $\times 100$ ) were calculated for each plant species and $C$. beticola isolate. Images of symptomatic leaf tissues were taken using a Nexus 5X (LGE, Seoul, South Korea) or iPhone 6 (Apple, Cupertino, CA) camera.

C. chenopodii pathogenicity to table beet. Two isolates of $C$. chenopodii, Lq15-006 and Lq16-002, were selected for pathogenicity tests on table beet (cultivar Ruby Queen). Inoculum production, inoculation, and growth conditions were as described above. Treatments in the replicated experiment included Lq15-006, Lq16-002, a $C$. beticola positive control (isolate Tb14-085), and a negative control solution $(0.01 \%$ [vol/vol] polysorbate-20). Each treatment contained five table beet plants, which were examined daily for symptom development over 4 weeks.

\section{Results}

Field surveys. Samples were selected from 10 symptomatic plant species for fungal isolations. Seventy-one cercosporoid isolates were morphologically identified from lesions collected from four plant species (Table 3). The majority of isolates were collected from leaf spots on lambsquarters $(75 \%)$, with the remainder isolated from eastern black nightshade (8\%), pigweed (Amaranthus retroflexus) (7\%), and spinach (10\%).

Phylogenetic species identification. The phylogenetic tree constructed using the aligned concatenation of five loci (act [186 bp] + $c m d A$ [216 bp] + hisH3 [321 bp] + ITS [454 bp] + tefl- $\alpha$ [281 bp]) identified seven isolates as $C$. beticola (Fig. 1). These $C$. beticola isolates originated from eastern black nightshade $(n=4)$, lambsquarters $(n=2)$, and spinach $(n=1)$. Fifty-one isolates clustered with $C$. chenopodii, and all originated from lambsquarters. $C$. chenopodii consisted of two separate clusters, consisting of four and 47 isolates, respectively. The remaining isolates clustered with C. cf. flagellaris $(n=11)$ and Cercospora sp. $\mathrm{G}(n=2)$ (Groenewald et al. 2013).

C. beticola pathogenicity assessment. During each experiment, all table beet plants inoculated with either $C$. beticola isolate developed CLS symptoms (Fig. 2), and the reisolation frequency of cercosporoid conidia was $100 \%$. Five of the 21 plant species inoculated with $C$. beticola exhibited $100 \%$ incidence of leaf lesions and reisolation frequencies of 60 to $100 \%$ (Table 2). These consisted of dock weed, lambsquarters, safflower, spinach, and wild mustard (Brassica kaber) (Fig. 2). Disease incidence ranged from 10 to $30 \%$ in broadleaf plantain (Plantago major), eastern black nightshade, pigweed, and snap bean (Phaseolus vulgaris) following inoculation with the two $C$. beticola isolates.

Nine plant species exhibited marginal leaf necrosis: namely, broadleaf plantain, buckwheat (Fagopyrum esculentum), carrot (Daucus carota subsp. sativus), eastern black nightshade, lettuce, lucerne (Medicago sativa), rye (Secale cereale), snap bean, and watermelon (Citrullus vulgaris var. lanatus). This symptom was also observed on control plants and therefore the pathogenicity of $C$. beticola to these species was discounted. However, reisolation from $C$. beticola inoculated plants indicated that cercosporoid conidia were present on the necrotic tissue at frequencies of 20 to $100 \%$. Stromata were also observed on leaf lesions and marginal leaf necroses.

Celery (Apium graveolens var. secalinum), pea (Pisum sativum), ragweed (Ambrosia artemisiifolia), sweet potato (Ipomoea batatas), velvetleaf (Abutilon theophrasti), and yellow nutsedge (Cyperus esculentus) did not exhibit disease symptoms after inoculation with either $C$. beticola isolate.

Natural senescence of leaves (distinct from lesion or marginal necrosis) occurred for both inoculated and control treatments over the 4-week period following inoculation. Cercosporoid conidia and stromata were observed on the naturally senescing tissues of each asymptomatic plant species inoculated with $C$. beticola. No sporulation was observed on necrotic or naturally senescent tissue of control plants.

C. chenopodii pathogenicity to table beet. Inoculation of table beet with $C$. chenopodii isolates Lq15-006 and Lq16-002 failed to result in disease, whereas inoculation with $C$. beticola isolate Tb14-085 induced typical CLS symptoms. Inoculum of each $C$. chenopodii isolate had $>90 \%$ viability on water agar.

\section{Discussion}

This study utilized two fungal isolates, identified as $C$. beticola using five loci (Vaghefi et al. 2018), and pathogenicity testing on plant species common to table beet fields in New York to re-evaluate host range. $C$. beticola was pathogenic to five of the 21 plant species tested. Although artificial inoculations provided indications of the pathogenicity of $C$. beticola, information on occurrence and persistence in natural field environments is required to determine the relative contribution of these alternative hosts to CLS epidemics. Field surveys of leaf lesions occurring on plants other than B. vulgaris subsp. vulgaris in table beet fields resulted in the identification of seven $C$. beticola isolates from lambsquarters, eastern black nightshade, and spinach. The majority of isolates $(72 \%)$ were identified as $C$. chenopodii on lambsquarters. These findings imply that $C$. beticola does not frequently cause lesions on weeds in table beet fields and challenge the concept that symptomatic weeds are important sources of inoculum for CLS epidemics.

C. beticola was pathogenic on dock weed, lambsquarters, safflower, spinach, and wild mustard following artificial inoculation in a controlled environment. These findings were supported by the isolation of $C$. beticola from lambsquarters and spinach in the field, albeit at low frequencies. A low incidence of pathogenicity to celery, lettuce, sweet potato, watermelon, and broadleaf plantain is in contrast to the results of Vestal (1933); however, direct comparisons are challenging because of the different origins of isolates and flux in $C$. beticola taxonomy. This study also provides the first report of $C$. beticola being pathogenic to dock weed and wild mustard.

Table 3. Plant species from table beet fields in 2016 exhibiting leaf lesions and the number of lesions yielding cercosporoid conidia

\begin{tabular}{llcc}
\hline $\begin{array}{l}\text { Common } \\
\text { name }\end{array}$ & \multicolumn{1}{c}{ Species } & $\begin{array}{c}\text { Symptomatic } \\
\text { plants sampled }\end{array}$ & $\begin{array}{c}\text { Cercosporoid } \\
\text { isolations }\end{array}$ \\
\hline $\begin{array}{l}\text { Eastern black } \\
\text { nightshade }\end{array}$ & Solanum ptycanthum & 6 & 6 \\
$\begin{array}{l}\text { Lambsquarters } \\
\text { Pigweed }\end{array}$ & $\begin{array}{l}\text { Chenopodium album } \\
\text { Amaranthus palmeri }\end{array}$ & 120 & 53 \\
Spinach & Spinacea oleracea & 7 & 5 \\
\hline
\end{tabular}


These two weed species were introduced to the United States and are reported to be widespread across the northern states, including New York (U.S. Department of Agriculture 2018). To date, there have been no reports of $C$. beticola being isolated from lesions on these plants in the field; however, targeted surveys are required to investigate their occurrence. The isolation of $C$. beticola from eastern black nightshade in the field, along with low disease incidence after artificial inoculation, also suggests that this species could harbor $C$. beticola inoculum.

The pathogenicity of $C$. beticola has been similarly reported on lambsquarters (Vestal 1933), safflower (Lartey et al. 2005), and spinach (Vestal 1933) in artificial inoculation trials; however the role of

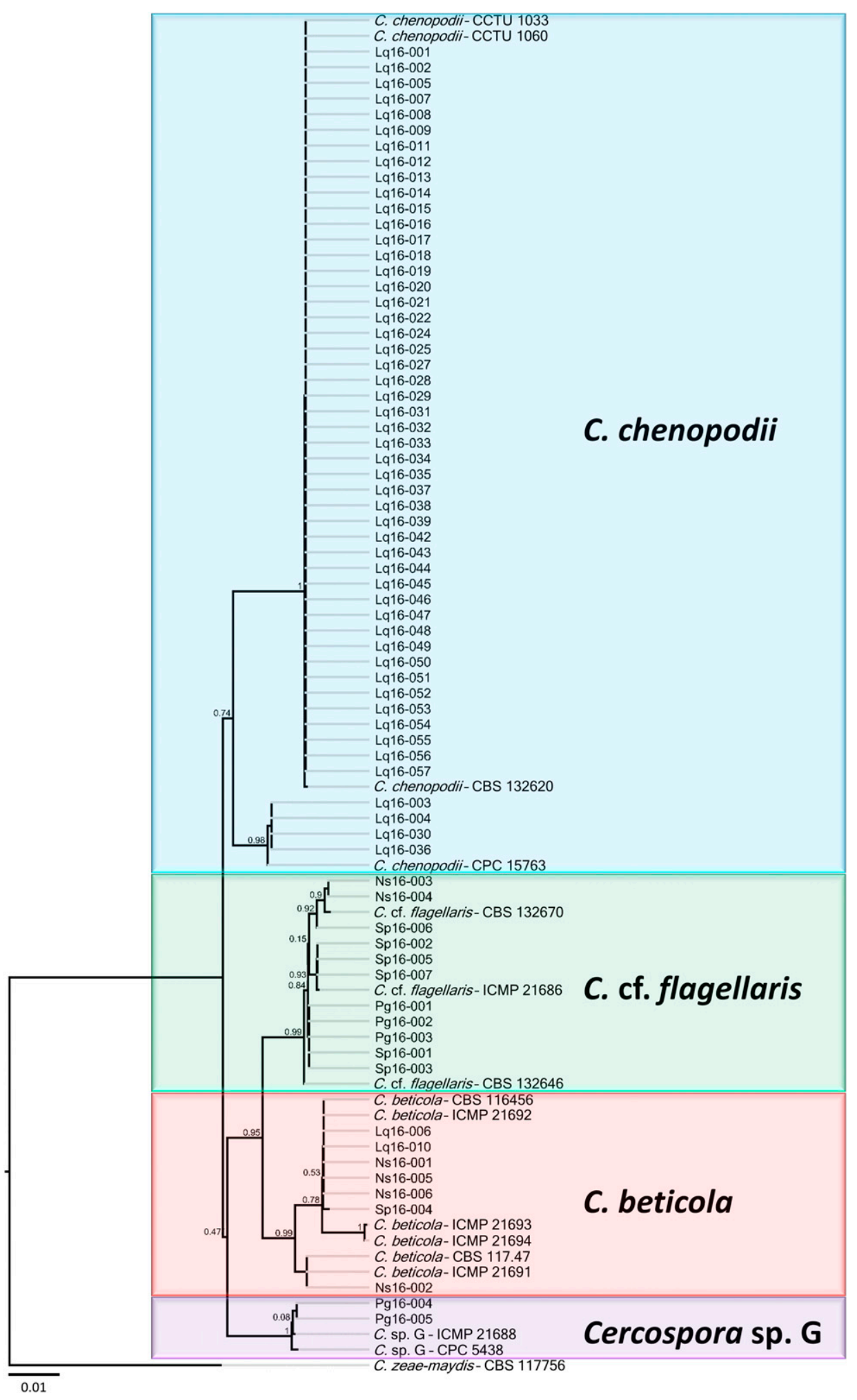

Fig. 1. Phylogenetic tree constructed using an approximately maximum likelihood analysis of the concatenated alignment of five loci (partial sequences of actin, calmodulin, histone, and translation elongation factor $1-\alpha$ and the internal transcribed spacer region). Four species of Cercospora were identified, including $C$. beticola, $C$. chenopodii, C. cf. flagellaris, and Cercospora sp. G. The tree was rooted to C. zeae-maydis (CBS 117756). Node support values were computed using the Shimodaira-Hasegawa test in the FastTree version 2.1.5 plug-in in Geneious version 11.1.5. The scale bar represents the number of estimated changes per position. 
these plants in the CLS disease cycle is not well understood. For example, although safflower exhibits lesions during artificial $C$. beticola inoculation trials, the fungus has yet to be isolated from this host in the field (Groenewald et al. 2006). In contrast, lambsquarters is commonly found growing within table beet fields of New York and frequently exhibits lesions during CLS epidemics on table beets (U.S. Department of Agriculture 2018; Vaghefi et al. 2017). However, the identification of predominantly $C$. chenopodii from lesions on lambsquarters, in the current study and by Vaghefi et al. (2017), conflicts with the conventional understanding that $C$. beticola causes the leaf spots on both table beet and lambsquarters (Jacobsen and Franc 2009). C. chenopodii has been similarly isolated from lambsquarters in Iran (Bakhshi et al. 2015a) and is considered as a fungal bioherbicide for lambsquarters (Scheepens and van Zon 1982). $C$. chenopodii isolated from lambsquarters was not pathogenic on table beet in artificial inoculation trials. These findings suggest that CLS on lambsquarters may occur in the same field as CLS of table beet but is initiated by different Cercospora spp.

The identification of $C$. cf. flagellaris and Cercospora sp. G from lesions on eastern black nightshade, pigweed, and spinach further demonstrates the challenge of identifying $C$. beticola-associated lesions on alternative hosts in the field. Vaghefi et al. (2018) investigated the pathogenicity of $C$. apii, C. cf. flagellaris, Cercospora sp. G, and C. zebrina on table beet. C. apii caused typical CLS symptoms, $C$. cf. flagellaris caused infrequent disease or was not pathogenic, and Cercospora sp. $\mathrm{G}$ and $C$. zebrina were also not pathogenic. More surveys of lesions in table beet-associated fields, during both table beet production and rotations, are required to elucidate the role of alternative hosts in CLS epidemics.

Infrequent isolation of $C$. beticola from lesions on lambsquarters, eastern black nightshade, and spinach in the field may indicate that $C$. beticola does not readily cause lesions on nonhost species. However,
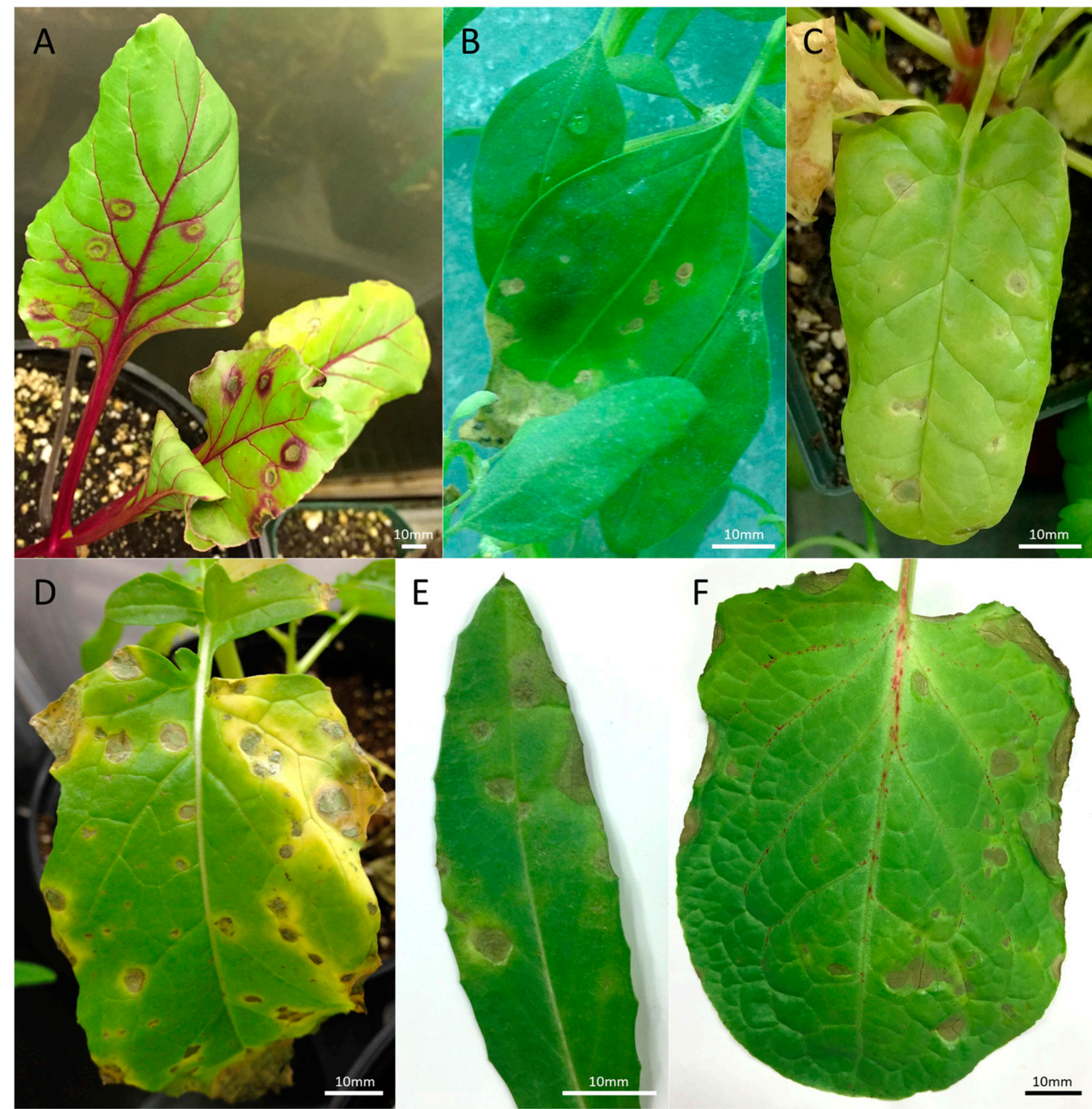

E

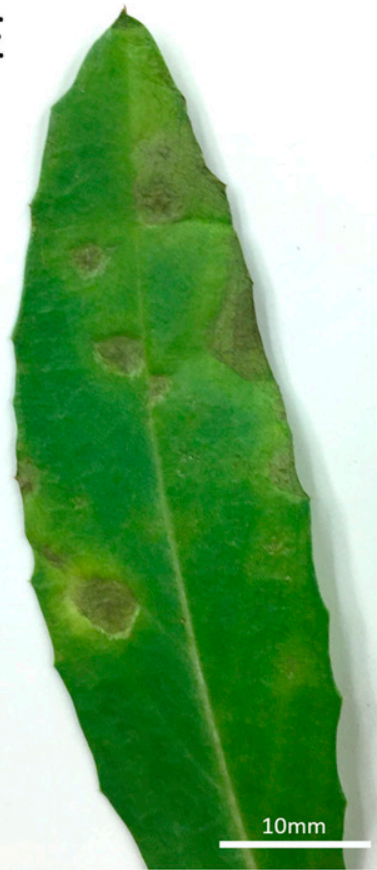

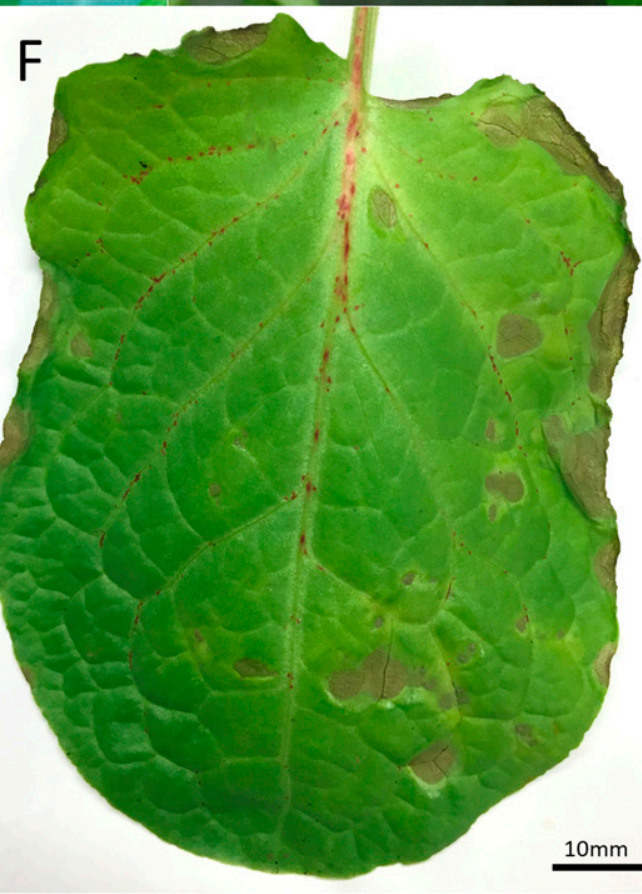

Fig. 2. Lesion formation on A, table beet (Beta vulgaris subsp. vulgaris 'Ruby Queen'), B, lambsquarters (Chenopodium album), C, spinach (Spinacia oleracea 'Bloomsdale'), D, wild mustard (Brassica kaber), E, safflower (Carthamus tinctorius 'Centennial'), and F, dock weed (Rumex obtusifolius) after inoculation with Cercospora beticola. 
a lack of lesions may not necessarily indicate the absence of $C$. beticola. During artificial inoculation experiments, $C$. beticola conidia and conidiophores were reisolated from necrotic and naturally senescing leaf tissues of several plant species that did not exhibit necrotic spots. Furthermore, a low incidence (10 to 30\%) of disease resulted in broadleaf plantain, eastern black nightshade, pigweed, and snap bean after artificial inoculation. Although $C$. beticola may have been weakly pathogenic on these species, it is also possible that other factors (e.g., insects, fungi, or physiological reactions) may have played a role in the lesion formation, with $C$. beticola subsequently colonizing the senescing tissue. This finding suggests that, under favorable environmental conditions such as high humidity and temperatures near $25^{\circ} \mathrm{C}, \mathrm{C}$. beticola may be able to survive saprophytically. Vestal (1933) proposed that $C$. beticola may live as a saprophyte after finding growth on dead leaves of plants other than sugar beet, including dandelion, lettuce, and potato. The survival of $C$. beticola on plant debris for 10 to 22 months (Khan et al. 2008) supports the important role of a saprophytic lifestyle for $C$. beticola.

The concept of plant pathogenic fungi surviving on nonhost plants was also proposed by Crous and Groenewald (2005). They outlined the "pogo stick hypothesis," which states that "host-specific fungal plant pathogens frequently exhibit the ability to colonize nonhost tissue or other substrates, forming fruiting bodies that will produce a limited amount of propagules, and enabling them to disperse further, in an attempt to find the host on which they are pathogenic" (Crous and Groenewald 2005). The potential for $C$. beticola to colonize senescing tissue of nonhost plants in the field, including lesions caused by other pathogens, needs to be considered. The methods of fungal isolation in this study were limited by the requirement for lesions with sporulating fungi. Thus, when a mixture of sporulating fungi was present on a lesion or C. beticola was not sporulating but present, $C$. beticola may not have been identified. Detection of $C$. beticola in a range of biological substrates, including alternative hosts, would benefit from improved species-specific detection tools such as quantitative PCR.

Informative host range studies require the detection and isolation of pathogens from field samples along with controlled inoculation experiments, with the selection of plant species for inoculation guided by field results. The infrequent isolation of $C$. beticola from lesions of nonhost plants in the field limited the objective selection of plants species for artificial inoculations. However, lesion formation on five plant species, along with $C$. beticola sporulation on senescing tissues of nonhosts, indicates that a broad range of substrates may play a role in $C$. beticola survival in a field environment. The potential involvement of multiple inoculum substrates in the field would explain the continued challenge of CLS control using the recommended 3-year rotation (Pethybridge et al. 2018).

\section{Acknowledgments}

We thank Carol Bowden, Traci Hoogland, Elizabeth Maloney, Sean Murphy, Alex Silva, and David Strickland (listed alphabetically by surname) for excellent technical support, and growers and gardeners for access to their fields and plots for sample collection.

\section{Literature Cited}

Bakhshi, M., Arzanlou, M., Babai-Ahari, A., Groenewald, J. Z., Braun, U., and Crous, P. W. 2015a. Application of the consolidated species concept to Cercospora spp. from Iran. Persoonia 34:65-86.

Bakhshi, M., Arzanlou, M., Babai-Ahari, A., Groenewald, J. Z., and Crous, P. W. 2015b. Is morphology in Cercospora a reliable reflection of generic affinity? Phytotaxa 213:22-34.

Bakhshi, M., Arzanlou, M., Babai-ahari, A., Groenewald, J. Z., and Crous, P. W. 2018. Novel primers improve species delimitation in Cercospora. IMA Fungus 9:299-332

Bobev, S. G., Groenewald, J. Z., and Crous, P. W. 2009. First report of Cercospora beticola as a pathogen of German statice (Goniolimon tataricum) in Bulgaria. Plant Dis. 93:553.

Cai, L., Giraud, T., Zhang, N., Begerow, D., Cai, G., and Shivas, R. G. 2011. The evolution of species concepts and species recognition criteria in plant pathogenic fungi. Fungal Divers. 50:121-133.

Chupp, C. 1954. A Monograph of the Fungus Genus Cercospora. Charles Chupp, Ithaca, NY.
Crous, P. W., and Groenewald, J. Z. 2005. Hosts, species and genotypes: Opinions versus data. Australas. Plant Pathol. 34:463-470.

Crous, P. W., Hawksworth, D. L., and Wingfield, M. J. 2015. Identifying and naming plant-pathogenic fungi: Past, present, and future. Annu. Rev. Phytopathol. 53:247-267.

Groenewald, J. Z., Nakashima, C., Nishikawa, J., Shin, H. D., Park, J. H., Jama, A. N., Groenewald, M., Braun, U., and Crous, P. W. 2013. Species concepts in Cercospora: Spotting the weeds among the roses. Stud. Mycol. 75:115-170.

Groenewald, M., Groenewald, J. Z., Braun, U., and Crous, P. W. 2006. Host range of Cercospora apii and $C$. beticola and description of $C$. apiicola, a novel species from celery. Mycologia 98:275-285.

Groenewald, M., Groenewald, J. Z., and Crous, P. W. 2005. Distinct species exist within the Cercospora apii morphotype. Phytopathology 95:951-959.

Hotegni Houessou, J. H., Beed, F., Sikirou, R., and Ezin, V. 2011. First report of Cercospora beticola on lettuce (Lactuca sativa) in Benin. New Dis. Rep. $23: 16$.

Jacobsen, B. J., and Franc, G. D. 2009. Cercospora leaf spot. Pages 7-10 in: Compendium of Beet Diseases and Pests. R. M. Harveson, L. E. Hanson, and G. L. Hein, eds. American Phytopathological Society, St. Paul, MN.

Katoh, K., Misawa, K., Kuma, K. i., and Miyata, T. 2002. MAFFT: A novel method for rapid multiple sequence alignment based on fast Fourier transform. Nucleic Acids Res. 30:3059-3066.

Katoh, K., and Standley, D. M. 2013. MAFFT Multiple Sequence Alignment Software Version 7: Improvements in performance and usability. Mol. Biol. Evol. 30:772-780.

Kearse, M., Moir, R., Wilson, A., Stones-Havas, S., Cheung, M., Sturrock, S., Buxton, S., Cooper, A., Markowitz, S., Duran, C., Thierer, T., Ashton, B., Meintjes, P., and Drummond, A. 2012. Geneious Basic: An integrated and extendable desktop software platform for the organization and analysis of sequence data. Bioinformatics 28:1647-1649.

Khan, J., Rio, L. E., Nelson, R., Rivera-Varas, V., Secor, G. A., and Khan, M. F. R. 2008. Survival, dispersal, and primary infection site for Cercospora beticola in sugar beet. Plant Dis. 92:741-745.

Knight, N. L., Vaghefi, N., Hansen, Z. R., Kikkert, J. R., and Pethybridge, S. J. 2018. Temporal genetic differentiation of Cercospora beticola populations in New York table beet fields. Plant Dis. 102:2074-2082.

Lartey, R., Caesar-TonThat, T., Caesar, A., Shelver, W., Sol, N., and Bergman, J. 2005. Safflower: A new host of Cercospora beticola. Plant Dis. 89:797-801.

McKay, M. B., and Pool, V. W. 1918. Field studies of Cercospora beticola. Phytopathology 8:119-136.

Miller, P. M. 1955. V-8 juice agar as a general purpose medium for fungi and bacteria. Phytopathology 45:461-462.

Pethybridge, S., Kikkert, J., Hanson, L., and Nelson, S. 2018. Challenges and prospects for building resilient disease management strategies and tactics for the New York table beet industry. Agronomy (Basel) 8:112.

Pool, V. W., and McKay, M. 1916. Climatic conditions as related to Cercospora beticola. J. Agric. Res. 6:21-60.

Price, M. N., Dehal, P. S., and Arkin, A. P. 2009. FastTree: Computing large minimum evolution trees with profiles instead of a distance matrix. Mol. Biol. Evol. 26:1641-1650.

Price, M. N., Dehal, P. S., and Arkin, A. P. 2010. FastTree 2 - Approximately maximum-likelihood trees for large alignments. PLoS One 5:e9490.

Rooney-Latham, S., Scheck, H. J., and Walber, T. M. 2011. First report of Cercospora beticola causing a leaf spot disease on Acanthus mollis in California. Plant Dis. 95:224.

Scheepens, P. C., and van Zon, H. C. J. 1982. Microbial herbicides. Pages 623-641 in: Microbial and Viral Pesticides. E. Kurstak, ed. Marcel Dekker, New York, NY.

Secor, G. A., and Rivera, V. V. 2012. Fungicide resistance assays for fungal plant pathogens. Pages 385-392 in: Plant Fungal Pathogens: Methods and Protocols. Bolton, M. D., and Thomma, B. P. H. J., eds. Humana Press, Totowa, NJ.

Shimodaira, H., and Hasegawa, M. 1999. Multiple comparisons of log-likelihoods with applications to phylogenetic inference. Mol. Biol. Evol. 16:1114.

Solel, Z. 1970. Survival of Cercospora beticola, the causal agent of sugar beet leaf spot, in Israel. Trans. Br. Mycol. Soc. 54:504-506.

U.S. Department of Agriculture. 2018. PLANTS Database. Natural Resources Conservation Service, United States Department of Agriculture. https://plants. usda.gov/

Vaghefi, N., Kikkert, J. R., Hay, F. S., Carver, G. D., Koenick, L. B., Bolton, M. D., Hanson, L. E., Secor, G. A., and Pethybridge, S. J. 2018. Cryptic diversity, pathogenicity, and evolutionary species boundaries in Cercospora populations associated with Cercospora leaf spot of Beta vulgaris. Fungal Biol. 122: 264-282.

Vaghefi, N., Nelson, S. C., Kikkert, J. R., and Pethybridge, S. J. 2017. Genetic structure of Cercospora beticola populations on Beta vulgaris in New York and Hawaii. Sci. Rep. 7:1726.

Vestal, E. F. 1933. Pathogenicity, host response and control of Cercospora leaf-spot of sugar beets. Iowa Agric. Exp. Stn. Res. Bull. 168:43-72.

Weiland, J., and Koch, G. 2004. Sugarbeet leaf spot disease (Cercospora beticola Sacc.). Mol. Plant Pathol. 5:157-166.

Zhou, J., Deckard, E. L., and Messersmith, C. G. 2005. Factors affecting eastern black nightshade (Solanum ptycanthum) seed germination. Weed Sci. 53: 651-656. 